\title{
“... ESSA COISA COMEÇOU A PEGAR!" - VIVÊNCIAS COTIDIANAS DE HOMENS OBESOS
}

\author{
“... This thing got me!”- Everyday life of obese men \\ “...la cosa empezó a fijarse!” - vivencias del cotidiano de \\ hombres obesos
}

Artigo Original

\section{RESUMO}

Objetivo: Conhecer as vivências cotidianas de homens com obesidade mórbida. Métodos: Pesquisa com abordagem qualitativa. Foram entrevistados nove homens com diagnóstico de obesidade mórbida que estavam em seguimento no pré-operatório para cirurgia bariátrica, com idade de 27 a 50 anos, casados, com renda familiar de dois a três salários mínimos, caso de obesidade na família e hipertensão como comorbidade. A investigação ocorreu no período de maio a setembro de 2012 em um hospital de referência no tratamento à obesidade e realização de cirurgia bariátrica em Fortaleza, Ceará, Brasil. Realizaram-se entrevistas semiestruturadas com análise, através da Análise de Conteúdo, do tipo análise temática. Eis as categorias de análise definidas: tornar-se obeso, vivências cotidianas do homem obeso e cirurgia bariátrica - um caminho possível para um cotidiano com saúde. Resultados: Os principais achados relacionaram-se a uma rotina diária fixa, com reduzido espaço para atividade física e de lazer. A obesidade, para $90 \%$ dos entrevistados, existe desde a infância e trouxe dificuldade na realização das ocupações no dia a dia. Esses homens optaram pela cirurgia por não perderem peso com dieta, exercício e medicamento, em razão da permanência de problemas de saúde, bem como dificuldades nas relações afetivas e no trabalho. Conclusão: Identificouse a demanda de uma reorganização das vivências cotidianas dos homens para que haja uma efetiva ação da cirurgia bariátrica, a fim de que os usuários sejam protagonistas dessa modificação, inserindo atividades saudáveis e contínuas no seu cotidiano.

Descritores: Obesidade Mórbida; Homens; Cirurgia Bariátrica; Atividades Cotidianas.

\section{ABSTRACT}

Objective: To understand the everyday life of men with morbid obesity. Methods: Qualitative research. Interviews were carried out with nine men diagnosed with morbid obesity in the preoperative period for bariatric surgery. They were 27-50 years old, married, had a household income of two/three minimum wages, history of obesity in the family and hypertension as a comorbidity. The research was conducted from May to September 2012 in a reference hospital for the treatment of obesity and bariatric surgery in Fortaleza, Ceará, Brazil. Semi-structured interviews were carried out and data underwent Content Analysis and thematic analysis. The categories for analysis were: becoming obese; everyday life of obese man and bariatric surgery - a possible path to a healthy everyday life. Results: The main findings are related to a fixed daily routine, with reduced space for physical activity and leisure. Obesity is present since childhood in $90 \%$ of the respondents, and it has brought difficulties in performing daily activities. These men chose the surgery because they have not been able to lose weight with diet, exercise and medicines, because of continuing health problems as well as difficulties in personal relationships and at work. Conclusion: It was identified a demand for a reorganization of the everyday life of men so that there is an effective action of the bariatric surgery to provide users with an opportunity to be protagonists of this change by including healthy and ongoing activities in their everyday life.

Descriptors: Obesity, Morbid; Men; Bariatric Surgery; Activities of Daily Living.

Recebido em: 23/12/2015

Revisado em: 26/02/2016

Aceito em: 03/03/2016 


\section{RESUMEN}

Objetivo: Conocer las vivencias del cotidiano de hombres con obesidad mórbida. Métodos: Investigación de abordaje cualitativo. Se entrevistaron nueve hombres con el diagnóstico de obesidad mórbida en seguimiento del preoperatorio para la cirugía bariátrica con edad entre los 27 y 50 años, casados, con renta familiar de dos a tres sueldos mínimos, caso de obesidad en la familia e hipertensión. La investigación se dio en el periodo entre mayo y septiembre de 2012 en un hospital de referencia para el tratamiento de la obesidad y realización de cirugía bariátrica de Fortaleza, Ceará, Brasil. Se realizaron entrevistas semiestruturadas con el análisis a través del Análisis de Contenido del tipo análisis temático. Las categorías de análisis definidas fueron: tornarse obeso, vivencias del cotidiano del hombre obeso y cirugía bariátrica - un camino posible para un cotidiano con salud. Resultados: Los principales hallazgos se relacionaron con una rutina diaria fija y reducido tiempo para actividad fisica $y$ de ocio. La obesidad existe desde la infancia para el 90\% de los entrevistados y ha dejado la dificultad en realizar las actividades del cotidiano. Esos hombres han decidido por la cirugía porque no perdían peso con la dieta, el ejercicio y el medicamento, en razón de la permanencia de los problemas de salud así como las dificultades en las relaciones afectivas y en el trabajo. Conclusión: Se identificó la necesidad de una reorganización de las vivencias del cotidiano de los hombres para una efectiva acción de la cirugía bariátrica para que los usuarios sean los protagonistas de esa modificación incluyendo actividades saludables y continuas en su cotidiano.

Descriptores: Obesidad Mórbida; Hombres; Cirugía Bariátrica; Actividades Cotidianas.

\section{INTRODUÇÃO}

No Nordeste, a cultura do homem viril está atrelada ao simbolismo de Lampião ("cabra da peste"), que se associa à noção de valentia, de sujeito na lida diária com a terra, projetando nos homens atuais a falta de preocupação com a beleza ou aparência, pois sua função é prover a família. A obesidade masculina, muitas vezes, está relacionada aos conceitos culturais de cada país ${ }^{(1)}$.

Esses processos socioculturais são constituídos nas vivências cotidianas, compreendidas como espaço de formação do sujeito, que se inicia no nascimento e, com a imersão no universo cultural, vai se legitimando. Essas vivências são parte da existência de todo e qualquer ser humano. Nessa dimensão social, o homem vai se apropriando da linguagem, dos objetos culturais, dos costumes e valores que contribuem para a constituição do ser, permitindo sua relação com o outro e a sociedade ${ }^{(2)}$. Esse movimento das experiências do cotidiano é moldado pela motivação do sujeito - o ser cotidiano se edifica do singular para o plural.
Entender o cotidiano implica compreender a relação sujeitocotidiano-história-sociedade na dialética constante da vida, considerando que as modificações ocorrem de acordo com o contexto do vivido ${ }^{(3)}$.

No século XIX, a adiposidade corporal era sinal de status e riqueza; entretanto, a obesidade não era desejada por todos, apenas indicava prestígio social ${ }^{(4)}$. O autor mostra em seu estudo que a diferença fundamental da contemporaneidade em relação ao século XIX é que, atualmente, o mínimo sinal de gordura é rechaçado. Além disso, os referenciais de obesidade e magreza mudaram com o tempo. Hoje, o corpo aceitável é absolutamente magro, longilíneo, com padrão estético, tendente ao belo. Alguns aspectos podem contribuir para essas modificações dos padrões de beleza na sociedade atual, como as tecnologias em saúde, a medicina estética e dermatológica, a moda, a gastronomia e a alimentação diária ${ }^{(5)}$.

Em razão desse fenômeno do culto ao belo e do cuidado com o corpo, a obesidade está em ascensão mundial. Com efeito, dados epidemiológicos apontam que a população brasileira possui uma prevalência de excesso de peso de $52 \%$ e $18 \%$ com obesidade. Ao comparar essa situação de saúde entre homens e mulheres, eles denotam maior risco para obesidade, tendo-se registrado excesso de peso em $56,5 \%$ e $49,1 \%$ da população, respectivamente ${ }^{(6)}$.

No enfrentamento da obesidade, as estratégias em saúde são organizadas desde a atenção básica, com foco na promoção da saúde, até a alta complexidade, com o tratamento cirúrgico ${ }^{(7)}$.

Posto nesse âmbito, é necessária uma reflexão sobre gênero e saúde masculinos, entretanto, o estudo do homem nas ações de saúde precisa redimensionar a visão e as práticas de saúde, pois tanto homens como mulheres devem ser cuidados de maneira integral e equitativamente. No tocante à relação obesidade e homem, o sexo masculino é visto como fator de risco de mortalidade para cirurgia bariátrica $^{(8)}$.

Ante a complexidade do homem, da obesidade, da cirurgia bariátrica e sua relação com o cotidiano, o objetivo do estudo foi conhecer as vivências cotidianas de homens com obesidade mórbida.

\section{MÉTODOS}

Durante os meses de maio a setembro de 2012, desenvolveu-se esta pesquisa, com abordagem qualitativa, em um hospital de referência no tratamento à obesidade e realização de cirurgia bariátrica, em Fortaleza, Ceará, metrópole com 2.452.185 habitantes.

Os usuários desse serviço provêem, principalmente, do Município de Fortaleza e demais comunidades do Estado do Ceará, encaminhados pelas unidades básicas de saúde. 
Participaram do estudo homens com diagnóstico de obesidade mórbida (Índice de Massa Corporal - IMC acima ou igual a $40 \mathrm{~kg} / \mathrm{m}^{2}$ ), idade superior a 18 anos, que estavam no pré-operatório de cirurgia bariátrica. Foram excluídos da investigação os usuários que abandonaram o seguimento por qualquer motivo, tendo-se recusado a realizar os procedimentos pré-cirúrgicos, e homens que durante a fase pré-operatória desenvolveram algum tipo de complicação/ contraindicação para realizar a cirurgia bariátrica.

Nove homens participaram do estudo, sendo esse número definido por meio da saturação teórica de informações ${ }^{(9)}$. Realizaram-se entrevistas semiestruturadas, compostas por perguntas abertas e fechadas, versando sobre o objeto de estudo. A entrevista conteve duas etapas, a primeira foi constituída por perguntas fechadas, que forneceram as informações objetivas para a caracterização dos sujeitos, como informações clínicas e sociodemográficas. A segunda parte do instrumento constituiu dos eixos temáticos norteadores: conte-me quando percebeu que começou a engordar; descreva sua relação com a família e o trabalho; (re)lembre alguma dificuldade no desempenho de atividade cotidiana.

Inicialmente, foi realizada uma reunião com a equipe responsável pela cirurgia bariátrica do hospital para apresentação da pesquisa e seleção dos sujeitos a investigar, conforme os critérios definidos. Os pacientes foram contatados no ambulatório, no dia da consulta médica.

Optou-se por gravar e transcrever as informações coletadas na entrevista e seu conteúdo foi organizado e classificado de acordo com a compreensão do discurso do sujeito, para extração dos tópicos de análise. Utilizou-se da Análise de Conteúdo do tipo análise temática ${ }^{(10)}$. Durante o exame das informações, realizou-se a primeira fase (pré-análise), na qual se definiram os núcleos temáticos a considerar no estudo; em seguida, efetuou-se a exploração do material, momento em que emergiram cinco temáticas das falas dos informantes-chave, das quais três foram eleitas como mais relevantes - tornar-se obeso, vivências cotidianas do homem obeso e cirurgia bariátrica, sendo apreciadas pelos nove informantes. As outras duas temáticas, trabalho e acesso ao serviço de saúde, foram excluídas desta análise. Na última fase, procedeu-se à interpretação, que se baseou no referencial teórico acerca da obesidade mórbida ${ }^{(11)}$, homem $^{(12)}$ e cotidiano ${ }^{(2)}$ na visão antropológica e da saúde coletiva. Analisou-se o conteúdo e discutiu-se a segunda parte do instrumento, aspectos relacionados a vivências cotidianas, como lazer, família, autocuidado, fatores que levaram a realizar a cirurgia, com suporte, no qual foram definidas as seguintes categorias: tornar-se obeso; vivências cotidianas do homem obeso; cirurgia bariátrica - um caminho possível para um cotidiano com saúde.
As falas dos participantes estão identificadas por siglas alfanuméricas (Homem Obeso - HO1, e assim sucessivamente), para preservar o anonimato, conforme Resolução 466/12 do Conselho Nacional de Saúde, Ministério da Saúde, Brasil.

A pesquisa recebeu o parecer de aprovação $n^{\circ} 31729$ do Comitê de Ética em Pesquisa da Universidade Fortaleza.

\section{RESULTADOS E DISCUSSÃO}

Os sujeitos do estudo se constituíram em nove homens com faixa etária de 27 a 50 anos, raça parda, a maioria era casado e com ensino médio completo. Sete deles exerciam as ocupações de professor, comerciante, segurança, chefe de serviço geral, multimídia, e um estava desempregado. A renda familiar dos sete que trabalhavam era de dois a três salários mínimos. Quatro informantes apresentaram casos de obesidade na família (pais, filho ou esposa); sete moravam em casa própria e dois em cômodo alugados; cinco eram católicos, um era evangélico, dois eram espíritas e um não referiu religião. Todos tinham IMC acima de 40 $\mathrm{kg}$ e relataram comorbidade de hipertensão autorreferida.

Os participantes exprimiram características próprias da obesidade mórbida com critérios para cirurgia bariátrica - IMC $>40 \mathrm{~kg} / \mathrm{m}^{2}$ - e comorbidades, como diabetes e hipertensão, que impactavam no seu cotidiano e saúde. Em razão disso, a trajetória percorrida até a cirurgia bariátrica está relacionada à perda de peso, com enfoque na retomada de uma vida mais saudável e com longevidade, demonstrando sofrimentos, dificuldades e expectativa da cirurgia bariátrica como a última opção ou chance para o emagrecimento - aspectos demonstrados nas falas dos informantes-chave.

\section{Tornar-se obeso}

A obesidade, para $90 \%$ dos entrevistados, existe desde a infância, com ingesta de alimentos hipercalóricos em virtude do cuidado alimentar das mães, ao vislumbrarem mais a quantidade do que a qualidade do alimento. É constatado o fato de que essas condições no início da vida influenciam a obesidade na fase adulta ${ }^{(13)}$. A vivência de ser uma pessoa com obesidade mórbida é constituída nas relações sociais, culturais e históricas, no campo individual e coletivo. Dessa forma, a visão sobre a obesidade é diferenciada de acordo com a cultura de cada sociedade ${ }^{(14)}$.

No imaginário de famílias nordestinas do interior, a gordura está associada a status social, beleza e saúde, dado significativo na fala de um entrevistado cuja mãe o associou a um "jumento preto", animal que no sertão traz a simbologia da força e resistência, diversidade do sertão nordestino brasileiro, e é ligado ao sagrado. A gordura como 
status e poder remete aos primórdios da história ${ }^{(15)}$, como se observa na seguinte fala:

"Nasci dentro de um restaurante. No interior, tem muito de dizer assim: 'meu filho gordo'. Isto, antigamente, 'meu filho gordo', era sinal que os pais tinham boas condições, tinha aquela visão que of filho gordo era uma honra para o pai." (HO7, 38 anos, funcionário público, residente no interior do estado).

Os pais sentiam orgulho de ter filhos "cheinhos", o que estava associado à condição econômica da família. A questão da obesidade infantil na representação social da cultura nordestina denota associação entre gordura e saúde, aspecto que pode contribuir para a obesidade na fase adulta $^{(2)}$. Tal situação proporciona consequências negativas, pois estudo aponta que o IMC acima dos parâmetros da saúde em crianças influencia no desenvolvimento da obesidade na fase adulta $^{(14)}$.

$\mathrm{Na}$ história da enfermidade ${ }^{(11)}$, relatada pelos informantes-chave, desde a infância, a alimentação está no centro da vida. Ressaltam-se a relevância da interferência da cultura sobre o alimento e as relações que permeiam a alimentação, como a escolha dos alimentos, tanto no âmbito individual como no coletivo(2). O comer e a comida fazem parte da elaboração de vida dos sujeitos ${ }^{(12)}$, pois todas as atividades, principalmente as de lazer, estão atreladas à alimentação, como sair no final de semana para a churrascaria ou promover encontros no domicílio, a cervejinha no final de semana, o tira-gosto etc, como relatado na seguinte fala:

"Como eu não bebia mais no bar, aí eu comecei a fazer em casa, à vontade, né? Fazia muito e em grande quantidade." (HO1, 50 anos, professor, residente na zona urbana).

Os entrevistados relataram a existência de um familiar com obesidade, seja mãe, pai, irmã, esposa ou filhos. Tal aspecto corrobora com achados de pesquisas de base genética ou hereditária ${ }^{(13)}$. Mas as causas da obesidade são multifatoriais. Além da fonte hereditária, pode-se pensar nas questões ambientais (referentes aos ambientes obesogênicos). Esses aspectos são descritos pelos informantes quando relatam suas vivências cotidianas, compreendidas como atividades realizadas no dia a dia, ações significativas que ensejam transformações, ligadas às atividades de vida diária (AVD) e ao desempenho ocupacional.

Considera-se cotidiano o espaço social onde a pessoa vive e se ocupa, não se tratando de mera rotina, repetição automática de ações e movimentos (fazer por fazer), mas um espaço próprio, onde o sujeito busca praticar atividades que possibilitem a criatividade, a transformação ${ }^{(3)}$ e a promoção de saúde ${ }^{(16)}$. Ressalta-se que uma rotina rígida, sem espaço para ocupações saudáveis (como a prática de atividade física), contendo apenas atividades ligadas ao ato de comer e trabalhar, pode ser um preditor da obesidade, como mostra a fala a seguir:

"Eu trabalho manhã, tarde e de noite. Eu quase não tenho tempo para mim mesmo." (HO1, 50 anos, professor, residente em Fortaleza).

A pessoa com obesidade necessita (re)organizar e (res) significar suas práticas cotidianas para (re)construir o seu dia a dia, com vistas ao enfretamento da obesidade ${ }^{(14)}$.

\section{Vivências cotidianas do homem obeso}

As vivências cotidianas do obeso necessitam de um olhar focal (no sujeito) e ampliado (no contexto vivido por ele), pois as experiências da cotidianidade do obeso podem ser a mola propulsora da enfermidade. Deve-se compreender a interligação do sujeito com seu contexto físico, cultural, social e religioso, ou seja, sua história de vida, hábitos, rotina diária e estilo de vida. Dessa maneira, pode-se ter uma real dimensão da influência das vivências cotidianas da pessoa.

O cotidiano daqueles que vivenciam a obesidade, muitas vezes, é fragmentado e reduzido ao ato de comer e à comida. Dessa maneira, desarticula e desorganiza suas outras relações sociais, afetivas e de trabalho, o que enseja dificuldade na realização das atividades da vida diária, como também restrição da participação em ocupações sociais, levando a um isolamento e ao surgimento de outras enfermidades ${ }^{(17)}$.

Esse aspecto é sensível nos informantes, quando ressaltam a complexidade no desempenho das ocupações diárias $^{(18,19)}$ e na mobilidade ${ }^{(20)}$ :

"Hoje eu acho muito desconfortável colocar e tirar calça jeans, preferencialmente. Amarrar sapato, sabe? Se eu tiver que fazer isto várias vezes ao dia, fico logo cansado, ai vou logo pra rede (risos)." (HO6, 35 anos, desempregado, residente na capital).

"Vixe, quando não deu para passar na catraca de ônibus." (HO2, 33 anos, comerciante, reside em Fortaleza).

A obesidade pode influenciar na privação da participação da pessoa nas ocupações de atividade cotidiana, como autocuidado, trabalho e lazer, ocupações que podem contribuir para a saúde e o bem-estar de uma população ${ }^{(21)}$.

O trabalho constituiu outra atividade prejudicada pela obesidade e ressaltada pelos participantes. Neste tópico, eles demonstraram duas vertentes - sustento $^{(22)}$ e estigma $^{(23)}$. O sustento para o homem está associado com sua posição de provedor da família ${ }^{(24)}$ :

"O que sustenta a minha família. Me traz o básico, o necessário." (HO7, 38 anos, funcionário público, reside no interior). 
Sob o prisma do estigma, na visão do mercado de trabalho, as pessoas obesas são improdutivas e, algumas vezes, demitidas com justificativas veladas, ou seja, não estão no perfil da empresa:

"Tem muitas empresas hoje que tem notoriamente um preconceito com as pessoas obesas.” (HO9, 27 anos, marketing multimídia, reside em Fortaleza).

O trabalho também pode ser um fator de risco para a obesidade ${ }^{(2)}$, pois, em decorrência das modificações tecnológicas, houve uma substituição do trabalho de gasto energético alto pelo trabalho mecânico/automatizado.

As pessoas obesas precisam reestruturar suas estratégias e táticas na busca da reorganização do cotidiano, eliminando a concepção de rotina fixa, compreendida como ocupações com sequências estabelecidas e não modificáveis ${ }^{(25,26)}$. É determinante na busca de sentido das orientações e procedimentos advindos pela equipe de saúde para o tratamento da obesidade identificar as ocupações que são gatilhos para o aumento de peso e atividades promotoras de saúde, e aprender a viver o seu cotidiano, que se encontra na história, na essência do sujeito. O homem é seu próprio cotidiano, o qual não se caracteriza pela repetição de atos, mas se trata de uma elaboração desse mundo por meio do seu conhecimento ${ }^{(2)}$.

\section{Cirurgia bariátrica - um caminho possível para um cotidiano com saúde}

As dificuldades no dia a dia levaram os homens a buscar a cirurgia bariátrica como fonte de emagrecimento e qualidade de vida, revelando preocupação com as questões de saúde, como as comorbidades - hipertensão, apneia do sono, disfunção erétil. Por conseguinte, são aspectos que interferem no seu cotidiano e nas relações sociais.

Dessa maneira, a representação da enfermidade para o homem nordestino ${ }^{(1)}$ proporciona um influxo no imaginário masculino, ao identificar a possibilidade da morte e a não participação da vida familiar, aspecto demonstrado por um dos entrevistados:

"Se o médico me disse que não morreria por causa da gordura, não ia fazer. Ele disse que posso morrer devido à gordura. Para cuidar das minhas filhas." (HO2; 33 anos, comerciante, residente na capital).

Como também a interferência na sexualidade masculina, pela disfunção erétil e o fato de não proporcionar prazer à parceira:

"Então essa coisa começou a pegar, ai os médicos disseram que era por conta da obesidade, e como diz o jargão: sexo é vida!" (HO1, 50 anos, professor, residente de Fortaleza).
No sentido atribuído pelos participantes, era desnecessária a preocupação com o corpo e a saúde, e passaram a centrar seus esforços no provento para manter sua família. Não perceberam, no entanto, com essa atitude, que a saúde foi colocada em segundo plano, corroborando com outra pesquisa ${ }^{(27)}$.

Identificou-se nas falas e nas manifestações não verbais dos pesquisados que a cirurgia bariátrica é a única estratégia para manutenção da saúde, trazendo nova perspectiva para a vida da pessoa obesa. Ela aparece como solução de todos os problemas. Após uma longa jornada de procedimentos sem os resultados esperados, está se torna a solução imediata e definitiva para se ter saúde e recuperar o tempo e as oportunidades perdidas:

"Agora ou eu corro atrás do prejuizo, do que eu realmente quero ou então se eu gosto de viver. Então eu tenho que correr atrás de um pouco de vida." (HO9, 27 anos, marketing multinivel, residente na capital).

\section{CONSIDERAÇÕES FINAIS}

A obesidade mórbida proporciona consequências significativas no cotidiano das pessoas. São marcas que vão se acumulando ao longo da trajetória de vida. Verificouse nos relatos que a enfermidade vem desde a infância e é percebida pelo homem quando influi no desempenho das atividades do dia a dia. Isso o conduz à busca da cirurgia bariátrica como solução para a perda de peso, o que, em outros procedimentos, não foi possível acontecer.

Ao desvelar a sua rotina diária, o homem identifica a robotização e a mecanização das atividades, muitas delas relacionadas ao trabalho que fica voltado para o sustento da família, o qual tem importante papel na tomada de decisão para a procura da cirurgia bariátrica. Esta surge como solução mágica para a resolução de todos os problemas de saúde dos homens, principalmente relativos ao trabalho, ou seja, à manutenção do sustento familiar.

Nessa perspectiva, a pesquisa da vida cotidiana de homens com obesidade mórbida, no tocante ao précirúrgico, constitui um espaço importante para análise de fatores determinantes no seu ambiente, trazendo à tona algumas reflexões sobre o resgate da cotidianidade e a importância da reorganização e ressignificação desse espaço para contribuição no enfrentamento da obesidade.

\section{REFERÊNCIAS}

1. Braide ASG, Nations M, Katz AM, Munguba MCS. Corpo e sociedade contemporânea: significados socioculturais da obesidade mórbida para homens nordestinos. In: Amorim RF, Catrib AMF, Jorge M 
$\mathrm{AB}$, organizadores. Olhares da saúde coletiva sobre o corpo. Fortaleza: Editora UECE; 2011. p.124-151.

2. Heller A. O cotidiano e a história. São Paulo: Paz e Terra; 2008.

3. Ferraço CE. Pesquisa com o cotidiano. Educ Socied. 2007;28(98):73-95.

4. Gonçalves CA. O "peso" de ser um gordo: um estudo antropológico sobre obesidade e gênero. Mneme. 2004;5(11):599-624.

5. Riegel F, Siqueira DS, Silva FG, Pai DD. Percepções de pacientes submetidos à cirurgia bariátrica: orientações pré-operatórias da equipe de enfermagem. Rev Enferm UFPI. 2014;3(3):53-7.

6. Ministério da Saúde (BR). Vigilância de fatores de risco e proteção para doenças crônicas por inquérito telefônico (Vigitel) [acesso em 2016 Mar 22]. Disponível em: http://portalsaude.saude.gov.br

7. Ministério da Saúde (BR). Organização regional da linha de cuidado do sobrepeso e da obesidade na rede de atenção à saúde das pessoas com doenças crônicas. Brasília: Ministério da Saúde; 2014 [acesso em 2016 Mar 22]. Disponível em: ecos-redenutri.bvs.br/tikidownload_file.php?fileId $=675$

8. Mancini MC, Cercato C. Preparo e cuidado préoperatórios em clínica e endocrinologia. In: Segal A, Franques ARM, organizadores. Atuação multidisciplinar na cirurgia bariátrica: a visão da COESAS. São Paulo: Miró Editoras; 2012. p. 53-66.

9. Fontanella BJB, Luchesi BM, Saidel MGB, Ricas J, Turato ER, Melo DG. Amostragem em pesquisas qualitativas: proposta de procedimentos para constatar saturação teórica. Cad Saúde Pública, 2011;27(2):38994.

10. Bardin L. Análise de conteúdo. Lisboa: Edições 70; 1977.

11. Marcelino LF, Patrício ZM. A complexidade da obesidade e o processo de viver após a cirurgia bariátrica: uma questão de saúde coletiva. Ciênc Saúde Coletiva, 2011;16(12):4767-76.

12. Couto MT, Romeu G. Homens, saúde e políticas públicas: a equidade de gênero em questão. Ciênc Saúde Coletiva. 2012;17(10):2569-78.

13. Néspoli N, Novaes JV, Rosa CM. O corpo na cultura: obesidade como doença, biopolítica e normalização. Desafios. 2015;1(2):149-68.

14. Li S, Chen W, Sun D, Fernandez C, Li J, Kelly T, He J, Krousel-Wood M, Whelton PK. Variability and rapid increase in body mass index during childhood are associated with adult obesity. Int J Epidemiol. 2015;44(6):1943-50.

15. Vigarello G. As metamorfoses do gordo: história da obesidade no Ocidente: da Idade Média ao século XX. Petrópolis: Vozes; 2012.

16. Heidemann ITSB. Promoção da saúde e qualidade de vida: concepções da carta de ottawa em produção científica. Ciênc Cuid Saúde. 2012;11(3):613-9.

17. Banks E, Lim L, Seubsman S, Bain C, Sleigh A. Relationship of obesity to physical activity, domestic activities, and sedentary behaviours: cross-sectional findings from a national cohort of over 70,000 Thai adults. BMC Public Health. 2011;11(762):1-14.

18. Cartelo DGS, Souza ACA, Silva M, Cruz DMC, Andrade VS. Estrutura da prática da terapia ocupacional: domínio e processo. Rev. Triangulo Mineiro. 2010; 3(2):57-147.

19. Associação Brasileira dos Terapeutas Ocupacionais - ABRATO. A terapia ocupacional e as atividades de vida diária, atividades instrumentais da vida diária e tecnologia assistiva. Fortaleza: ABRATO; 2011.

20. Menegoni F, Galli M, Tacchini E, Vismara L, Cavigioli M, Capodaglio P. Gender-specific effect of obesity on balance. Obesity (Silver Spring). 2009;17(10):1951-6.

21. Forhan MA, Law MC, Vrkljan BH, Taylor VH. the experience of participation in everyday occupations for adults with obesity. Can J Occup Ther. 2010;77(4):210 8.

22. Woleck A. O trabalho, a ocupação, e o emprego: uma perspectiva histórica [Internet]. Criciúma: Instituto Catarinense de Pós-Graduação [acesso em 2012 Out 25]. Disponível em: http://www.iesc.ufrj.br/cursos/ saudetrab/trabalho\%20ocupa\%E7\%E3o.pdf.

23. Mattos RS, Luz MT. Sobrevivendo ao estigma da gordura: um estudo socioantropológico sobre obesidade. Physis. 2009;19(2):489-507.

24. Freitas WMF, Silva ATMC, Coelho EAC, Guedes RN, Lucena KDT, Costa APT. Paternidade: responsabilidade social do homem no papel de provedor. Rev Saúde Pública. 2009;43(1):85-90.

25. Choi B, Dobson M, Schnall P, Garcia-Rivas J. 24hour work shifts, sedentary work, and obesity in male firefighters. Am J Ind Med 2016. Epub ahead of print

26. Jacobs K, Jacobs L. Dicionário de Terapia Ocupacional. São Paulo: Roca, 2006. 
27. Moreno CAS, Silva AM, Cecato JF, Bartholomeu D, Montiel JM. Caracterização das mudanças psicológicas ocasionada em indivíduos submetidos à cirurgia bariátrica. Encontro. 2011;14(20):99-116.
Endereço para correspondência:

Carminda Maria Goersch Fontenele Lamboglia

Universidade de Fortaleza - UNIFOR

Curso de Educação Física

Av. Washington Soares, 1321

Bairro: Edson Queiroz

CEP 60811-905 - Fortaleza - CE - Brasil

E-mail: carmindalamboglia@gmail.com 